Investigación

\title{
Currículum, evaluación y acreditación en la formación docente. Un análisis de los Planes Nacionales de Argentina
}

\author{
Curriculum, assessment, and accreditation in teacher \\ education programs. An analysis of National Plans in \\ Argentina
}

Cappellacci, Inés; Molinari, Andrea

itinerarios

educativos

la revista del INDI
Inés Cappellacci * icappellacci@gmail.com Universidad de Buenos Aires, Argentina Andrea Molinari ** andrea.molinari@unipe.edu.ar Universidad de Buenos Aires (UBA) y Universidad Pedagógica Nacional (UNIPE), Argentina

Itinerarios educativos

Universidad Nacional del Litoral, Argentina

ISSN: 1850-3853

ISSN-e: $2362-5554$

Periodicidad: Semestral

núm. 15, e0011, 2021

revistadelindi@fhuc.unl.edu.ar

Recepción: 15 Septiembre 2020

Aprobación: 02 Septiembre 2021

URL: http://portal.amelica.org/ameli/jatsRepo/582/5822678006/ index.html

DOI: https://doi.org/10.14409/ie.2021.15.e0011

\section{c) (i) (9)}

Esta obra está bajo una Licencia Creative Commons AtribuciónNoComercial-CompartirIgual 4.0 Internacional.
Resumen: Las políticas de formación docente en Argentina, desde la existencia del Instituto Nacional de Formación Docente, se expresan en tres planes nacionales desarrollados en distintas gestiones de gobierno nacional. Cada uno generó un conjunto de regulaciones que tuvieron como propósito la territorialización de estas políticas en la órbita provincial. Esta diversidad de etapas, contextos y definiciones, se transforma en un motivo para la indagación sobre las continuidades en estos planes, qué cambios existieron y cuáles fueron las dinámicas de instalación de las políticas que se asumieron. Este trabajo se inscribe en un proyecto de investigación de la Universidad Pedagógica Nacional y su objeto de análisis es la formación docente inicial a partir del estudio comparativo de las políticas curriculares, acreditación y evaluación integral. Este conjunto de medidas demanda altos niveles de concertación entre el estado nacional y las jurisdicciones argentinas, dado que cualquier cambio que se formule en el plano de los lineamientos nacionales compromete al conjunto de instituciones dependientes de los gobiernos provinciales. Aquí se presenta un análisis de los procesos de concertación federal, el papel que desempeña el estado nacional y las tensiones entre lo federal, lo provincial y lo institucional en relación a la formación docente inicial.

Palabras clave: formación docente, políticas curriculares, evaluación de la formación docente, sistema formador argentino.

Abstract: Since the creation of the National Institute for Teacher Education in Argentina, teacher education policies are revealed in three national plans developed in different national government administrations. Each one generated a set of regulations that aimed at adjusting these policies to each provincial context. This diversity of stages, contexts, and definitions leads to the need for an analysis of plans continuity, undergone changes, and the dynamics of the implementation of the defined policies. This work is part of a research project of Universidad Pedagógica Nacional of Argentina and its object of analysis is preschool teacher education through the comparative study of curricular policies, accreditation, and comprehensive assessment. These measures demand a constant compromise between the national state and Argentine jurisdictions, 


\begin{abstract}
since any change introduced into national guidelines affects every provincial institution. This article provides an analysis of the federal concertation processes, the role played by the national state, and the tensions between federal, provincial, and institutional scopes as regards preschool teacher education.
\end{abstract}

Keywords: teacher education, curricular policies, of teacher education assessment.

\title{
La creación de un nuevo organismo para la formación docente
}

La formación docente en Argentina ${ }^{1}$ presenta una historia de sucesivas reformas complejas, inconclusas y discontinuas. Desde su pasaje del nivel secundario al nivel superior en 1969 se sucedieron políticas específicas que no lograron ejecutarsecompletamente. Aun así, constituyen verdaderas reformas en términos de Viñao (2016), en la medida en que fueron intentos de transformación o cambio educativo impulsados desde los poderes públicos, más allá de que cada propuesta haya producido procesos de innovación o de renovación pedagógica y didáctica en las comunidades locales, con gran adhesión a los lineamientos políticos. Se destacan el proyecto Magisterio de Educación Básica (MEB), el Programa de Transformación de la Formación Docente (PTFD) y el proceso de reforma enmarcada en la Ley Federal de Educación (LFE). Constituyen antecedentes sobre los que se asienta la propuesta de formación docente vigente en Argentina.

En el año 2006, se aprueba la Ley de Educación Nacional (LEN) 26.206 que reemplaza a la LFE 24195 de 1993. Esto implicó la reorganización de la estructura académica del sistema educativo en cuatro niveles (Educación Inicial, Educación Primaria, Educación Secundaria y Educación Superior) y ocho modalidades, modificando la estructura establecida en la LFE. Con respecto a la formación docente, la LEN confirma la reconfiguración del nivel de Educación Superior establecida en la Ley 24.521, de Educación Superior, de 1995. Este nivel educativo comprende a universidades e institutos universitarios así como a institutos de educación superior (formación docente y formación técnico profesional). También determina una duración de cuatro años de la carrera de grado para la formación de los docentes de todos los niveles educativos. Esta definición pone en acción políticas curriculares para la elaboración de planes de estudios y de estrategias de desarrollo curricular.

En la misma línea, establece la creación del Instituto Nacional de Formación Docente (INFD) con el fin de rearticular la fragmentación resultante de la aplicación de la LFE. Lo define como el organismo responsable de planificar y ejecutar, de forma concertada, políticas de articulación del sistema de formación docente inicial y continua, además de fortalecer las relaciones del sistema de formación docente y los otros niveles del sistema educativo, aplicar las normas orientadas a la evaluación, autoevaluación y acreditación de instituciones y carreras, validez nacional de títulos y certificaciones (exceptuando a las universidades), promover políticas curriculares, entre otras atribuciones. Esto da inicio a un nuevo impulso de desarrollo y jerarquización de la formación docente. 


\section{El INFD y los Planes Nacionales de la Formación Docente}

A partir de la creación del INFD, las políticas de formación docente se expresan en planes nacionales que originan regulaciones y líneas de trabajo, generalmente de cuatro años, acompañando las gestiones y el programa político del gobierno nacional, en acuerdo con las jurisdicciones. La diversidad de etapas, contextos y definiciones, se transformó en un motivo para la indagación de la investigación ya referida, que caracteriza las continuidades, los cambios y las dinámicas de instalación de las políticas que asumieron estos planes.

Los ámbitos de discusión y concertación bajo la coordinación del INFD son la Mesa Federal del Nivel Superior y el Consejo Consultivo. La primera es un espacio de análisis y discusión integrado por los directores de Educación Superior de las jurisdicciones. El Consejo Consultivo, por su parte, es un órgano de asistencia y asesoramiento integrado por representantes del Ministerio de Educación, del Consejo Federal de Educación, del Consejo de Universidades, del sector académico, del sector sindical docente y de las asociaciones de educación privada. Ambos órganos deben sesionar periódicamente. Las agendas de la Mesa Federal dan cuenta de las prioridades políticas de cada momento y los temas incluidos en ellas van en línea con las acciones previstas en los Planes Nacionales. En relación con el Consejo Consultivo, se observa un funcionamiento periódico entre los años 2007 y 2015, con sesiones más frecuentes entre 2007 y 2012 y un abandono a partir del año 2015 que coincide con el cambio de gestión de gobierno. Estos planes nacionales, desde una perspectiva que analiza a las políticas públicas como acción gubernamental, resultan un marco racional de acciones que impone metas y plazos a la vez que plasman los acuerdos de carácter federal (Martínez Nogueira, 1997). Estas políticas nacionales, en un régimen federal como el de Argentina, requieren de medidas concertadas y de instrumentos codiseñados para su implementación dado que se desarrollan en los territorios provinciales, comprometiendo su autonomía, sus recursos económicos, técnicos e institucionales.

El Plan Nacional de Formación Docente 2007-2010 (Res. CFE No 23/07) que se complementa con dos normas, Objetivos y Acciones 2008 (Res. CFE No 46/08) y Objetivos y Acciones 2010-2011 (Res. CFE No 101/2010), se estructura en tres grandes áreas: Desarrollo Institucional, Desarrollo Curricular y Desarrollo Profesional que engloban diez problemas y diez estrategias de acción. Este plan se basa en las conclusiones del Estudio de la calidad y cantidad de oferta de la formación docente, investigación y capacitación en la Argentina: Informe final realizado por María Cristina Davini (2015). Para el área de Desarrollo Institucional, las estrategias se orientan al fortalecimiento de la identidad y la planificación del sistema, a la organización institucional y al desarrollo de políticas estudiantiles. Con respecto al área de Desarrollo Curricular, las estrategias van se proponen incorporar experiencias de innovación, asegurar el derecho a una formación de calidad de los futuros docentes y desarrollar procesos de monitoreo y evaluación de las propuestas curriculares. En cuanto al Desarrollo Profesional, las estrategias están orientadas a fortalecer las actividades de investigación, la formación de los formadores y acciones de formación continua de impacto en las escuelas. Por su parte, el Plan Nacional de Formación Docente 2012-2015 (Res. CFE N¹67/12), incluido más tarde en el Plan 
Nacional de Educación Obligatoria y Formación Docente 2012-2016 (Res. CFE $\mathrm{N}^{\circ} 188 / 12$ ), define seis líneas de trabajo: Planeamiento y desarrollo del Sistema Nacional de Formación Docente en el marco de la construcción federal; Evaluación integral de la formación docente; Fortalecimiento del desarrollo curricular; Fortalecimiento de la formación continua y la investigación; Fortalecimiento de las trayectorias y la participación de los estudiantes; y Consolidación de la formación pedagógica con recursos digitales. Estos planes tuvieron como propósito la territorialización de estas políticas en la órbita provincial.

Finalmente, ya en otra gestión de gobierno nacional, el Plan Nacional de Formación Docente 2016-2021 (Res. CFE N² 286/16) se estructura en cuatro principios: justicia educativa, valoración de los docentes, centralidad de la práctica y renovación de la enseñanza, que guían a tres objetivos (garantizar la cantidad de docentes con la titulación requerida; mejorar la calidad de la formación docente inicial; y mejorar las prácticas de los equipos directivos y docentes en ejercicio). Se despliegan acciones sin perspectiva de sistema formador en lugar de líneas de política educativa. Esta breve presentación permite observar que las estructuras de estos planes dan cuenta de las prioridades políticas reguladas para cada período. Mientras los dos primeros planes tienen como objetivo el fortalecimiento del sistema formador, del conjunto de sus instituciones y sus actores en términos de planeamiento, gestión, evaluación, formación y desarrollo profesional, el último de ellos - extiende su período de vigencia a seis años sin justificación - direcciona su foco hacia políticas de formación docente inicial y continua, diluyendo sus intenciones y su atención sobre el conjunto del sistema formador.

\section{Las politicas curriculares}

La reforma curricular de la formación docente que se produjo en el marco de la LEN suscitó profundas transformaciones, sobre todo en lo que refiere a la formación de maestros de nivel inicial y de nivel primario. Si bien el conjunto de políticas docentes en este período abarcó, además, aspectos organizacionalesinstitucionales y del sistema formador, el cambio curricular jugó un papel central en el proceso transformador, claramente impulsado por el Estado nacional como promotor principal de las políticas educativas (Dussel, 2000). A partir de la intensidad con la que apareció la política curricular - sustantiva en los dos primeros planes y permaneció casi ausente en el último- se puede inferir que la tarea de regular la formación docente del país a través de lo curricular fue una de las estrategias privilegiadas para ir garantizando homogeneidad y coherencia en el sistema formador. La instalación de los cuatro años de duración de la formación implicó una fuerte acción por parte del Estado para asegurar que en todas las jurisdicciones se cumpliese con este mandato de la LEN.

El estado del cual partía la formación docente inicial, evidenciado en los diagnósticos, se encontraba lejos del escenario construido hasta el año 2015. Para 2007, todas las provincias habían desarrollado cambios en los planes de estudio para adecuarlos a los lineamientos nacionales aprobados por la LFE, pero con resultados muy diversos. Casi la mitad de las provincias contaba con propuestas institucionales formuladas a partir de los acuerdos aprobados por el Consejo 
Federal de Educación, lo cual dio lugar a más de tres mil títulos en todo el país. Esto dificultaba la movilidad tanto de los estudiantes como de los egresados a la hora de desempeñarse como docentes.

En el año 2007, con la definición de Lineamientos Nacionales Curriculares (Res. CFE No 24/07), los distintos planes de estudio debieron organizarse en torno a tres campos básicos de conocimiento, la Formación General, la Formación Específica y la Formación en la Práctica Profesional. Cada uno de estos campos presenta propósitos específicos. El campo que presenta más novedades es el de las prácticas profesionales, que se incorpora desde el inicio de la formación, tanto en actividades de campo como en el aula del instituto formador y se incrementa progresivamente en prácticas docentes en las aulas, culminando en la Residencia pedagógica integral. Se concibe a este espacio como vertebrador de toda la formación (Ball y Forzani, 2009) y al incluirse desde el inicio de la carrera se propone superar el sentido aplicativo que suponen los modelos deductivos de formación, en los que primero se aprende la teoría para luego aplicarla. Este abordaje simultáneo de los tres campos, ubicando a las prácticas desde el principio, pone de relieve que el sentido de la formación se juega a lo largo de toda la carrera. Además, frente a la gran diversidad de planes y títulos se realiza, durante 2008, un intenso trabajo de ordenamiento de las titulaciones y de elaboración de los nuevos diseños curriculares de alcance jurisdiccional. Como resultado de este proceso, se consolidó en menos de trescientos títulos la oferta de formación docente para todos los niveles y modalidades, lo que formaliza el carácter jurisdiccional de los diseños curriculares de la formación docente (Molinari, 2017). Estas acciones fueron ejes principales de los planes nacionales de ese período.

Otro hito importante, es la aprobación del Marco Referencial de Capacidades Profesionales de la Formación Docente Inicial (Res. CFE No 337/18), que define treinta y seis capacidades profesionales como construcciones complejas de saberes y formas de acción que permiten comprender e intervenir en distintas situaciones educativas. Así planteadas presentan, al menos, dos interrogantes, uno de carácter pedagógico y otro de carácter político. Las capacidades generales propuestas son seis: dominar los saberes a enseñar; actuar de acuerdo con las características y diversos modos de aprender de los estudiantes; dirigir la enseñanza y gestionar la clase; intervenir en la dinámica grupal y organizar el trabajo escolar; intervenir en el escenario institucional y comunitario; y comprometerse con el propio proceso formativo. Parecieran estar más orientadas a los docentes en ejercicio que a los estudiantes en formación. No anticipa, esta norma, un mapa de progreso de las capacidades, un gradiente planteado por etapas que pudiese dar pistas a la formación inicial y permitiera, como se propone, poner en diálogo - integrador - los distintos campos de la formación. Si estas capacidades son planteadas como resultados, como estándares, allí aparece el problema político, porque cobrarían sentido si se constituyeran en metas de una evaluación estandarizada, propósito que no se enuncia explícitamente. Deja planteado el dilema de si se trata de un preciosismo pedagógico, inútil para pensar la formación inicial en clave de mejora de su propuesta o de una acción política velada asociada a la evaluación de la formación docente.

Resulta evidente que el foco de la primera etapa de los Planes Nacionales (2007-2010) estuvo puesto en la adecuación de los diseños curriculares para 
la formación docente de los niveles Inicial y Primario (por su duración menor a cuatro años). En el 2009, se advierte el trabajo sobre los diseños curriculares para la Educación Especial, la Educación Física y la Educación Artística. Estas carreras forman para todos los niveles educativos y el acuerdo en la formulación de criterios comunes y federales y conforman un hito en la historia de la construcción política federal, según reconoce el Plan 20122016. Y en 2010, se inicia el proceso de revisión y elaboración de nuevos diseños curriculares para los Profesorados de Educación Secundaria y Superior, previsto en el Plan 2010/2011. En estos casos el proceso de transformación curricular asume características diferentes, dada la responsabilidad compartida con las universidades en la formación de profesores. Así, la Secretaría de Políticas Universitarias y el INFD establecieron, de forma conjunta, criterios generales para la renovación de los diseños curriculares, a través del Proyecto de Mejora para la Formación Inicial de Profesores para el Nivel Secundario. Se trató de un trabajo articulado y participativo de profesores de las universidades nacionales y de los institutos de formación docente que se plasmó en una serie de documentos para que funcionaran como insumos para la discusión y producción curricular de cada una de las provincias y de las universidades. El trabajo se organizó en torno a tres preguntas: ¿que\# es lo que importa que los futuros docentes comprendan del campo disciplinar?, ¿que\# tipo de experiencias debería transitar un futuro profesor durante su formación? y ¿cómo sabemos, tanto los formadores de profesores como los estudiantes del profesorado, que están construyendo comprensión?

El Plan 2012-2015, recuperando acciones de años anteriores, puso el foco en completar la modificación de los diseños curriculares para la educación secundaria y la educación superior y se establecieron acuerdos para la estructura de los diseños curriculares jurisdiccionales de todas las carreras de formación docente y la formulación o la actualización de los diseños curriculares para la formación docente de la educación secundaria y de la educación superior.

A su vez, en el Plan 2016-2020, dentro del punto 2, «Mejorar la calidad de la formación inicial», el INFD reconoce los logros y los avances de la política de desarrollo curricular, sin embargo, afirma que «existen desafíos pendientes en el sistema formador y en los conocimientos, las capacidades y las actitudes de los egresados». Estos desafíos se orientan a la saldar las diferencias entre las jurisdicciones, los ámbitos estatal y privado, y las instituciones, y agrega «pese a la reciente reformulación de los diseños curriculares, los nuevos docentes no siempre cuentan con las capacidades para ejercer una profesión que se ha vuelto cada vez más exigente» (7-8). Este diagnóstico supondrá una serie de propuestas entre las cuales se destaca consolidar los procesos de desarrollo curricular y de validez nacional de títulos.

En este punto, se enfatiza la importancia de la reformulación periódica de los diseños curriculares y refuerza la idea de que el INFD es el organismo responsable de aplicar los criterios acordados federalmente referidos a la validez nacional de títulos y certificaciones de estudio. Sin embargo, han sido escasas las acciones de acompañamiento y de revisión de los diseños curriculares. Y en cierta medida, se verifica un debilitamiento de los procedimientos de validez nacional de los títulos y de los diseños curriculares jurisdiccionales, en la última etapa de este período. 


\section{Las politicas de evaluación y acreditación}

Entre las funciones del INFD se encuentra la evaluación del sistema formador, plasmadas en el Plan Nacional de Formación Docente 2007-2010. Allí se parte del problema la insuficiente sistematización, seguimiento y evaluación curricular como base para su mejora y actualización, y se propone como objetivo adecuar criterios sobre validez nacional y homologación de títulos y certificaciones. En materia de evaluación y acreditación se desarrollan dispositivos que paulatinamente consolidan prácticas de participación técnico políticas de carácter federal en lo que respecta al monitoreo de los planes de estudio y sus desarrollos. La creación de la Comisión Federal de Evaluación (2008), la Evaluación del Desarrollo Curricular (2011), la Evaluación de estudiantes (2013), el Operativo Enseñar (2017) y la creación de la Comisión Nacional de Evaluación y Acreditación de la Calidad de la formación docente (2018) constituyen políticas que tienden a consolidar mecanismos de evaluación en el sistema formador. Cada dispositivo recupera el sentido político del plan nacional en el que se enmarca.

\section{La Comisión Federal de Evaluación}

Desde 2007, la validez nacional de los títulos ocupó gran parte de las discusiones en las mesas técnicas y políticas, y definió nuevas regulaciones sobre los procedimientos de validez de títulos y certificaciones al crear a la Comisión Federal de Evaluación (CoFEv). En la Res. No 2170/08 se establece que para otorgar la validez nacional se requiere el cumplimiento de una serie de requisitos y la elaboración de un informe técnico a cargo de la CoFEv, otorgando la validez nacional hasta cinco cohortes. Esta medida impulsa a las jurisdicciones a elaborar actualizaciones periódicas de los diseños curriculares jurisdiccionales que además suman las condiciones institucionales, el régimen orgánico y el régimen académico, a los criterios de evaluación.

Está previsto que la Comisión esté integrada por cinco representantes técnicos - uno por cada región del país - especialistas en currículum y formación docente, designados por el INFD a propuesta de las jurisdicciones. Esta conformación puede asumirse como un indicador de la decisión de consolidar la participación permanente de las jurisdicciones en las políticas del nivel, sobre todo si se atiende al historial de excepciones iniciadas a partir de la transferencia de los servicios educativos nacionales a las provincias en temas de validez. Este proceso que comienza en 1996 con un régimen de equivalencia de títulos y de estudios de validez nacional (decreto 1276/96 y sucesivos decretos hasta el año 2005) se interrumpe en vías de un ordenamiento e incorpora, por primera vez, una instancia de representación regional de carácter técnico que se propone garantizar ciertos estándares nacionales e intenta débilmente romper con la inercia de la «descentralización centralizada» parafraseando a Filmus (1997). 


\section{La evaluación del desarrollo curricular}

Entre las políticas de evaluación y acreditación, en 2011 se puso en marcha el proceso de evaluación para las carreras de formación docente para Educación Inicial y Educación Primaria y en 2014 para las carreras de Educación Especial, Educación Artística y Educación Física. La elaboración del dispositivo de evaluación se realizó de manera concertada políticamente en el marco de las Mesas Federales y del Consejo Consultivo. Cada provincia conformó un equipo de trabajo que producía conjuntamente con el equipo técnico nacional los sentidos políticos de la evaluación, la batería de instrumentos y llevaba adelante localmente la implementación junto con los equipos directivos, docentes y estudiantes de los ISFD. Incluyó también la participación de pares evaluadores de otras instituciones para que dieran garantías de transparencia al proceso. Se evidencia en la propuesta las intenciones de federalización de la política en tanto contempla la incorporación de la mayor cantidad y diversidad de actores locales en las distintas instancias de diseño, implementación y análisis de los resultados. En los Informes relevados se señala que fueron evaluados 522 institutos de formación docente de gestión estatal y privada, 61 carreras de Educación Inicial y 228 carreras de Educación Primaria. Respecto de la evaluación de las carreras de Educación Especial, Educación Artística y Educación Física, participaron 353 institutos de gestión estatal y privada.

La evaluación recortó su objeto de estudio en la realización práctica de los planes de estudio e implicó tanto la evaluación de los propios textos curriculares como de los procesos a través de los cuales las propuestas formativas se concretaron efectivamente en la práctica, centrando su atención en el currículum real. Abordó los aspectos de la organización institucional que inciden en el desarrollo curricular, las prácticas formativas de los profesores, tanto en el ámbito institucional como en el de las aulas y las trayectorias estudiantiles. El dispositivo se organizó en cuatro etapas. La primera se orientó a la autoevaluación del desarrollo curricular para la que se conforma la Comisión interna con representantes de docentes, estudiantes, equipo directivo y referente de evaluación de la institución y se elabora un informe integrado del instituto formador. La segunda consistió en una visita metaevaluativa de la comisión de observadores externos para elaborar recomendaciones. En la tercera etapa se elaboró un informe de carácter jurisdiccional y en la última, las conclusiones finales en un Informe Federal.

\section{La evaluación de estudiantes}

Entre los años 2013 y 2014 se llevó adelante el dispositivo de evaluación con alcance nacional que involucró a 10506 estudiantes de formación docente que se encontraban cursando la Práctica III en 513 carreras de Educación Inicial y de Educación Primaria ofertadas por 392 instituciones formadoras (estatales y privadas) de todas las jurisdicciones. Su metodología asumió una perspectiva investigativa de carácter exploratorio y se propuso, a la vez, como una experiencia formativa para todos los actores involucrados, transformando la experiencia en un elemento valioso para reflexionar sobre las prácticas de enseñanza y de aprendizaje en las instituciones formadoras. 
Dándole continuidad a las políticas de evaluación implementadas y configurando en ese momento una política integral de evaluación, esta evaluación incorporó en su dispositivo la estructura de gestión jurisdiccional y nacional desplegada ya en la Evaluación de Desarrollo Curricular. Este proceso replicó el criterio federal en la participación de las decisiones incorporando multiplicidad de actores para cada etapa.

Los ejes que se abordaron en este proceso fueron cuatro: 1) saberes, valores y capacidades en desarrollo sobre la escuela y el trabajo docente; 2) saberes, valores y capacidades en desarrollo sobre los procesos de enseñanza y los procesos de aprendizaje; 3 ) valoraciones sobre los aportes de la formación en diferentes campos y 4) los actores y las instituciones. La evaluación de los estudiantes se llevó a cabo en una jornada de trabajo en la institución y se desarrolló en tres momentos. El primero consistió en la resolución por escrito de consignas sobre un relato de clase presentado a parejas. Además del instrumento de evaluación, todos los momentos contaban con material orientador para los equipos que coordinaban la actividad. El segundo momento propuso un debate en torno a situaciones escolares presentadas en un video y requirió producción grupal y el último fue un cuestionario que debía completarse individualmente.

\section{ElEnseñar}

Con el cambio de gestión política, el INFD desarrolló otras líneas de evaluación, En 2017 implementó la evaluación diagnóstica Enseñar. Se destaca la formulación de los objetivos y de las perspectivas de esta nueva visión de la evaluación al analizar el documento marco de este operativo. En este documento, para demostrar que ningún sistema nacional y planificado era esquivo a su evaluación, se recuperó una selección de experiencias latinoamericanas. Todas ellas hacen foco en el ingreso y el egreso del nivel superior y en el ingreso a la carrera docente. La mayoría de los casos tienen un carácter selectivo y ninguno se refiere a la evaluación de políticas. El enfoque que prevalece en estos casos da cuenta de los modelos input-output y no de procesos. Este solapamiento de sentidos podría ser comprendido como parte de una estrategia que intentó instalar mecanismos de selección meritocrática inscriptos en discursos sobre la calidad educativa y la mejora de formación docente. Los ejes evaluados indagados pusieron el foco en los criterios pedagógicos y en una evaluación de comunicación escrita. Se llevó adelante en todo el país, con grandes manifestaciones de resistencia.

\section{La Comisión Nacional de Evaluación y Acreditación de la Calidad}

A fines de 2018, el Consejo Federal de Educación aprobó -en ausencia de cuatro provincias y con el rechazo de seis- la creación de la Comisión Nacional de Evaluación y Acreditación de la Calidad de la formación docente (CNEAC). Esta comisión se conformó con ocho académicos de reconocida trayectoria, tres integrantes del gobierno educativo nacional y un miembro móvil. Es notable el predominio de los miembros de la academia sobre los actores políticos en la composición de la Comisión, lo que podría expresar, desde 
las perspectivas tecnocráticas, el intento por mostrar la neutralidad de estos procesos. Si a este rasgo se le suma la ausencia de profesores - a través de la representación gremial - la evaluación y la acreditación podrían ser consideradas una mera cuestión técnica y, fundamentalmente, despolitizada. En su articulado se proponen las funciones de la CNEAC, entre las que se encuentran emitir dictámenes conducentes al otorgamiento de la validez nacional de los títulos y carreras de la formación docente, producidos por los instrumentos elaborados por la Secretaría de Evaluación Educativa. Esta definición, sumado a que quien coordine la CNEAC pueda ser un miembro del Ministerio de Educación de la Nación, deja al INFD en un plano secundario y al borde del incumplimiento respecto de las funciones que la LEN le atribuye. Hasta tanto no se elabore la reglamentación acerca de su funcionamiento y los modelos de evaluación que asumirá no puede anticiparse cuánto de construcción colectiva y participación federal contemplarán los dispositivos, pero habiendo transcurrido diez meses de su aprobación sin avances en esta línea puede inferirse que las objeciones planteadas por las centrales sindicales y algunas de las provincias paralizaron su tratamiento.

\section{A modo de cierre}

En las últimas cinco décadas, Argentina - como otros países de la regiónimplementó una serie de reformas orientadas a la jerarquización de la formación docente. Desde el pasaje al nivel terciario a fines de los años 60 hasta su reconocimiento como formación de educación superior a fines de los '90, las sucesivas reformas fueron ganando en extensión y en diversificación de su oferta. De todos modos, debe reconocerse que no son lineales ni tienen rasgos de continuidad, fundamentalmente, en lo relativo a sus contextos históricopolíticos. Desde la transición democrática en los años 80, las reformas han tenido intensos períodos de descentralización y de gran influencia de organismos internacionales que, aun bajo relaciones herméticas y de cúpulas (Torres, 2002; Ruiz, 2016), instalaron discursos globales conformando climas de época. La última de estas reformas, producida en el marco de la LEN, establece un hito con la creación de un organismo, dependiente del Ministerio de Educación Nacional, cuyo mandato es el diseño de políticas de formación docente de carácter federal. La LEN mantiene los tres niveles de gobierno y administración del sistema educativo y propone como ámbito de concertación de políticas al Consejo Federal de Educación -donde participan los gobiernos educativos de cada jurisdicción argentina, además de representantes del Consejo de universidades - Este Consejo tiene competencias para producir un tipo de normativa denominada resoluciones, que en la mayoría de los casos son de cumplimiento obligatorio para todo el país.

A partir de los análisis realizados a lo largo del trabajo se puede señalar que las políticas curriculares vinculadas a la formación docente en Argentina se sustentan en acuerdos federales establecidos en los planes nacionales, lo que implica que son refrendados por los ministros de educación jurisdiccionales. En los procesos de elaboración de los diseños curriculares, el trabajo del INFD y el de los equipos jurisdiccionales se estructuró en función del diagnóstico elaborado por Cristina Davini en 2005. Así establecen algunos criterios federales con el fin de garantizar 
la circulación de los docentes titulados a partir de la definición de la validez nacional de los títulos.

Otro aspecto sustantivo es la decisión de que los diseños curriculares sean jurisdiccionales y no institucionales, a la vez que se observa la definición escalonada de elaboración de los diseños curriculares a través de la definición de criterios comunes: la unificación de la duración de los profesorados, con la determinación de una carga horaria mínima de 2600 horas reloj y no menos de cuatro años académicos; la unificación de la denominación de los títulos docentes en todo el país; la homologación de los profesorados de las distintas jurisdicciones y los de la misma jurisdicción entre sí mediante la definición de una estructura curricular común, organizada en torno a tres campos formativos simultáneos: el de la Formación General, el de la Formación Específica y el de la Formación en la Práctica Profesional, entre otros aspectos. Este escalonamiento también se refleja en el inicio del trabajo con los documentos destinados a la formación de profesores para los niveles Inicial y Primario, que hasta 2008 eran carreras de menor cantidad de años y horas de estudios, para continuar con los de Educación Física, Educación Artística y Educación Especial y, en último término, la adecuación o creación de los diseños curriculares disciplinares para los niveles Secundario y Superior.

La instalación de mecanismos de evaluación y acreditación, como los aquí analizados, constituye una política que tiende a fortalecer al sistema formador, incorporando de forma paulatina prácticas de monitoreo sobre el propio desarrollo planteadas en clave de mejora. El circuito de producción de información con alta participación de los actores clave, tanto a nivel institucional como del gobierno del sistema formador, podría facilitar la toma de decisiones técnico-políticas locales y consolidar la articulación federal. Aunque estos avances no tengan un horizonte cercano en la consecución de esas metas dadas las diferencias y heterogeneidad jurisdiccionales.

A medida que se produce el avance de cada línea de trabajo, se destaca que disminuye el énfasis puesto en los diseños curriculares y aumenta el del desarrollo curricular y el de la evaluación. En el último Plan 2016-2021 no se observa un cambio rotundo en lo que refiere a los documentos elaborados, sin embargo, se desatendieron los criterios federales y se fomentó el avance de las definiciones institucionales en tanto que la garantía de la unidad de la formación podría sustentarse en los resultados de las evaluaciones de estudiantes de la formación docente. Esta última reflexión podría enmarcarse en lo que Filmus (2017) define como una de las estrategias de desarticulación de la capacidad del Estado para producir orientaciones generales y la de facilitar la no conducción del sistema educativo, a contrapelo de etapas anteriores.

Los análisis desarrollados en este trabajo sobre las políticas de formación docente en Argentina se proponen mostrar la centralidad que estas han tenido durante las últimas décadas y las veladas rupturas que se evidencian en el último período de gobierno. Mientras las escasas medidas asumidas entre 2015 y 2019 proponen, con énfasis muchas de ellas, dar continuidad a las políticas precedentes, en los propios desarrollos de las normas se encuentran sentidos y propósitos contrapuestos. Esto significa que no solo se interrumpieron políticas en desarrollo (como las de equipamiento o formación permanente) y se redujo la capacidad del INFD en el juego político, sino que se montaron sobre políticas 
sensibles para la historia de la formación docente (como las de evaluación y acreditación) sentidos opuestos a los que lograron la aceptación.

\section{Referencias}

Ball, D.; Forzani, F. (2015). The Work of Teaching and the Challenge for Teacher Education. Journal of Teacher Education 60(5) 497-511. 10.1177/0022487109348479 http://jte.sagepub.com

Davini, M. C. (2015). Estudio de la calidad y cantidad de oferta de la formación docente, investigación y capacitación en la Argentina: Informe final. Ministerio de Educación de la Nación.

Dussel, I. Tiramonti, G.; Birgin, A. (2000). Towards a new cartography of educational reform. Reflections on the educational decentralization in Argentina. Journal of Curriculum Studies, 32(04), 537-559.

Filmus, D. (1997). La descentralización educativa en Argentina: elementos para el análisis de un proceso abierto. Coloquio Regional sobre Descentralización de la Educación en América Central, Cuba y República Dominicana, 1-14.

Filmus, D. (Comp.) (2017). Educar para el mercado. Escuela, universidad y ciencia en tiempos de neoliberalismo. Octubre.

Filmus, D. (1997) La descentralización educativa en Argentina: elementos para el análisis de un proceso abierto. Coloquio Regional sobre Descentralización de la Educación en América Central, Cuba y República Dominicana (1997 Nov. 3-5: San José).-CLAD; Países Bajos. Gobierno Nacional; Costa Rica. Ministerio de Planificación Nacional y Política Económica, Costa Rica.

Martínez Nogueira, R. (1997). Análisis de Politicas Públicas. INAP.

Molinari, A. (2017). Las políticas curriculares de la formación docente a partir de la Ley de Educación Nacional. De los diseños al desarrollo curricular. En García, A.; López, S. y Marzioni, C. La formación docente en escenarios contemporáneos. Universidad Nacional de Quilmes.

Ruíz, G. (Comp.) (2016). La educación secundaria obligatoria en el marco de las reformas educativas nacionales. Eudeba.

Torres, R. M. (2002). Reformadores y docentes: el cambio atrapado entre dos lógicas. Educação na América Latina. UNESCO/OREALC.

Viñao, A. (2006). El éxito o fracaso de las reformas educativas: Condiciones, limitaciones y posibilidades. En Gimeno Sacristán, J. (Comp.). La reforma necesaria: entre la politica educativa y la práctica escolar. Morata.

\section{Notas}

1 Este trabajo es parte del informe parcial de investigación «La formación de maestras y maestros en Argentina en los inicios del siglo XXI. Dinámicas y tensiones en el campo de la formación docente inicial para los niveles inicial y primario», de la Universidad Pedagógica Nacional - Programación Científica 2020-2022.

\section{Notas de autor}

* Inés Cappellacci es licenciada en Ciencias de la Educación por la Facultad de Filosofía y Letras de la Universidad de Buenos Aires. Especialista en Especialista en Políticas Educativas y Magister en Ciencias Sociales con Orientación en Educación - FLACSO - Argentina. Profesora adjunta interina de las cátedras 
de Pedagogía y Problemas y Corrientes Pedagógicas Contemporáneas en la Facultad de Filosofía y Letras de la UBA y en el Departamento de Ciencias Sociales y Educación de la Universidad Pedagógica Nacional. Desarrolla tareas de investigación en ambas universidades. Trabaja temas de formación docente.

** Andrea Molinari es licenciada en Ciencias de la Educación por la Facultad de Filosofía y Letras de la Universidad de Buenos Aires. Especialista en Metodología de la Investigación Científica por la Universidad Nacional de Lanús. Profesora regular de las cátedras de Didáctica General en la Facultad de Psicología de la UBA y en el Departamento de Ciencias Sociales y Educación de la Universidad Pedagógica Nacional. Desarrolla tareas de investigación en ambas universidades como directora y co-directora de proyectos. Trabaja temas de formación docente. 